A - Research concept and design

B - Collection and/or assembly of data

$\mathrm{C}$ - Data analysis and interpretation

D - Writing the article

E - Critical revision of the article

F - Final approval of article

\section{A review of the effect of a Walker ankle-foot orthosis on gait biomechanics in healthy individuals}

\author{
Karol Lann Vel Lace*A-F (D), Michalina Błażkiewicz ${ }^{A-F}$ (D) \\ Faculty of Rehabilitation, Józef Piłsudski University of Physical Education \\ in Warsaw, Poland
}

*Correspondence: Karol Lann Vel Lace; Józef Piłsudski University of Physical Education, Faculty of Rehabilitation, Poland; email: lannkarol@gmail.com

\begin{abstract}
Walker orthoses are one of the most popular substitutes to regular cast. They are used by people who have experienced acute trauma to the ankle complex. This review aimed to summarize and update information on the currently published research explicitly related to the application of ankle-foot orthoses and to help improve the understanding of how orthoses influence gait pattern, in particular the kinematics, kinetics, and muscle activity parameters. A review of papers published from 1999 to 2019 was conducted. The databases searched included PubMed Central, PubMed, ScienceDirect and EBSCO, a total of 546 articles were found. The review is based on 11 articles that were selected for further analysis. Kinematics parameters were described in 8 papers, kinetics parameters in 6 papers, the effect of a Walker orthosis on muscle activity in 2 papers, and the effects of a Walker boot on energy cost, pain and balance were discussed in 3 papers. A Walker orthosis significantly reduces ankle joint motion and significantly reduces lower leg muscle activity. On the other hand, it may cause secondary site pain due to leg length differences and increase in energy expenditure. A Walker orthosis is a useful tool replacing total contact casts and allowing earlier weight bearing and rehabilitation. It is important to use it for the shortest possible period of time and to always use compensation of leg length discrepancy.
\end{abstract}

Keywords: kinematics, kinetics, foot

\section{Introduction}

An orthosis is a device worn on the body that is intended to support the patient by partially taking over the function of the damaged part of the locomotor apparatus. Orthoses can be divided into mass-produced and custom-made that are specially prepared for the client and individually selected for them. They are used on any part of the body, starting from the neck [1], through the torso [2], upper limbs [3] and ending with the lower limbs [4].
There are many types of ankle orthoses with different functions or used in different cases. Orthotics can be used for people with both neurological and orthopedic problems [5]. Walker boots are used by people who have experienced acute trauma to the ankle complex such as fractures, severe ankle sprains and patients who have undergone surgery in this area [6]. What is more, Walker orthoses may be prescribed to patients as part of conservative treatment or cast substitute after surgical treatment of Achilles tendon rupture. The other group of patients who benefit from the ability to ambulate in

This is an Open Access journal, all articles are distributed under the terms of the Creative Commons Attribution-NonCommercial-ShareAlike 4.0 International (CC BY-NC-SA 4.0). License (http://creativecommons. org/licenses/by-nc-sa/4.0/). 
a Walker orthosis are those who suffer from ulcers due todiabetes mellitius [7].The main function of an orthosis is to relieve and protect the diseased tissue, limit the range of motion in the joint and enable gait by helping reduce pain [8]. This type of orthosis can be used as a substitute for a conventional cast or as a continuation of treatment after its removal. Compared to a classic cast, a Walker boot has many advantages: it is lighter, which translates into less impact on the biomechanics of gait and can be removed for exercise, it allows for supervision of the healing process and tissue hygiene $[6,9]$. Low-cut Walkers are used when the metatarsal $[10,11]$ or tarsal areas [12] require immobilization. High-cut Walkers are used in the above cases and when the treatment concerns the ankle or muscles located in this area [6]. Certain high Walker boots are adjustable in the sagittal plane of movement in the upper ankle joint. For this purpose, the orthosis uses a special hinge [6].

A Walker orthosis has many advantages, but it causes differences in the length of the limbs. It was shown that simulated leg-length discrepancy causes asymmetrical limb loading and that a greater loading rate and a greater proportion of the weight bearing load is sustained by the shorter limb [13]. Leg length discrepancy can lead to plantar aponeuritis [14], and degenerative changes in the knee joints [15] as well as hip and lumbar spine [16]. Depending on the kind of trauma, immobilization with the use of an orthosis can last for several weeks, during which the patient increasingly loads the limb in the orthosis. In some studies, the reduction in lower leg muscle activity was shown to be more effective in Walker than in fiberglass casts [17]. Akizuki, Gartman [18] found that a Walker boot reduces triceps surae activity by $21 \%$ and by $43 \%$ if a one 1 inch heel wedge is added. In other articles, it has been shown that the metabolic energy expenditure of gait with immobilized ankle joint increases by $9-15 \%$ for walking with the same speed in comparison to normal gait. Energy expenditure while walking in cast increases the energy expenditure by $26 \%$ [19]. Therefore, the aim of this paper was to review the research investigating kinematics, kinetics and muscle activity parameters during walking with an ankle-foot orthosis - Walker to help improve our understanding of how this type of orthoses influence gait pattern.

\section{Materials and methods}

\section{Search strategy}

The review was limited to studies analyzing gait biomechanics in a Walker orthosis. The electronic search of databases was performed on 28th July 2019.
The articles were limited to those published from January 1999 to July 2019. The PubMed Central, PubMed, ScienceDirect and EBSCO databases were searched to identify appropriate literature using the search terms: (("Walker orthosis") OR ("Walking boot") OR ("Walking boot") OR ("Walker boot") OR ("step ankle orthosis")) AND ("gait" OR "locomotion" OR "kinetics" OR "kinematics").

\section{Eligibility}

Only full-text articles were selected from the electronic databases. The inclusion criteria were (1) the full-text of the publication in English or Polish, (2) assessment of kinetic or kinematic gait parameters; (3) analysis of muscle activity and other parameters relating to gait, (4) analysis of gait in low-cut or highcut Walker orthoses, (5) studies including only healthy participants. Exclusion criteria were (1) the number of participants below 5, (2) no Walker orthosis used in the study, (3) only plantar pressure distribution analysis in the study, (4) articles evaluating the biomechanics of gait in orthoses other than Walker, (5) papers in which disabled individuals were examined. Titles, abstracts and full-texts of the retrieved documents were sequentially reviewed by two independent authors (KL and $\mathrm{MB}$ ) to determine their relevance to the topic. Also, the reference lists of all the studies included for the review were searched manually for their additional relevance. Moreover, the manuscripts which lacked basic information about the equipment or the characteristics of the study group were also excluded. No restriction was applied regarding sex, age and type of Walker orthosis.

\section{Review process}

Duplicate articles were rejected. The title and abstract for the selected articles were first screened according to the eligibility criteria. Furthermore, the fulltext evaluation was performed if the title and abstract could not provide adequate information for the article screening process. Rejected articles were re-screened to avoid misinterpretation. The titles, abstracts and then full-text of the papers identified by the search were screened by two independent reviewers (the authors: $\mathrm{KL}$ and $\mathrm{MB}$ ) to choose those that met the selection criteria and extract the data. Decisions about which trials should be selected were made by negotiation. One reviewer (KL) compiled all articles in using a Mendeley Reference Manager software. Next, the articles that had been found and approved were divided into four subgroups depending on whether they evaluated (1) kinematic parameters, (2) kinetic parameters, (3) EMG, (4) energy expenditure, balance and pain. 


\section{Results}

Initially, the electronic database screening process yielded 546 articles. The screening of titles and abstracts enabled to single out 497 articles, and an agreement was reached for 21 articles, which were identified to be related to the aim of the literature survey. Following the eligibility criterion of full-text studies, 11 articles were selected for the review. No articles were retrieved from the reference lists, yielding a total of 11 articles for the review process (Fig. 1).

\section{Kinematic parameters}

Kinematic parameters during walking in different walker boots were described in 8 papers (Tab. 1). In five of these articles, kinetic parameters were also characterized. In two papers, the metabolic energy cost of walking with the immobilized ankles was compared to normal walking, while only one paper discussed muscle activity.
Kinematic gait analysis was performed in eight articles. In six articles, motion capture analysis systems were used, whereas in two papers $[8,20]$, the authors used just an electronic walkway. In five articles [9,19,21-23], the study samples were rather small (below fifteen participants), which may have affected the results and limited the generalization capabilities. In two studies $[8,20]$, the authors compared different kinds of braces, but did not compare them to barefoot walking or walking in street shoes, which makes it impossible to assess the effect of these orthoses on gait. Only one study analyzed the effect of heel wedges on gait kinematics [23].

In conclusion, walking velocity decreases to $13 \%$ while wearing an orthosis, but some papers failed to provide precise data. The analysis of temporo-spatial characteristics of gait in Walker compared to normal gait demonstrated that the step length of the limb in Walker drops to $4 \%$ whereas for the limb without Walker, this value falls to $7 \%$. The step width rises to $15 \%$ and therefore the gait cycle time increases to $9 \%$ [24].

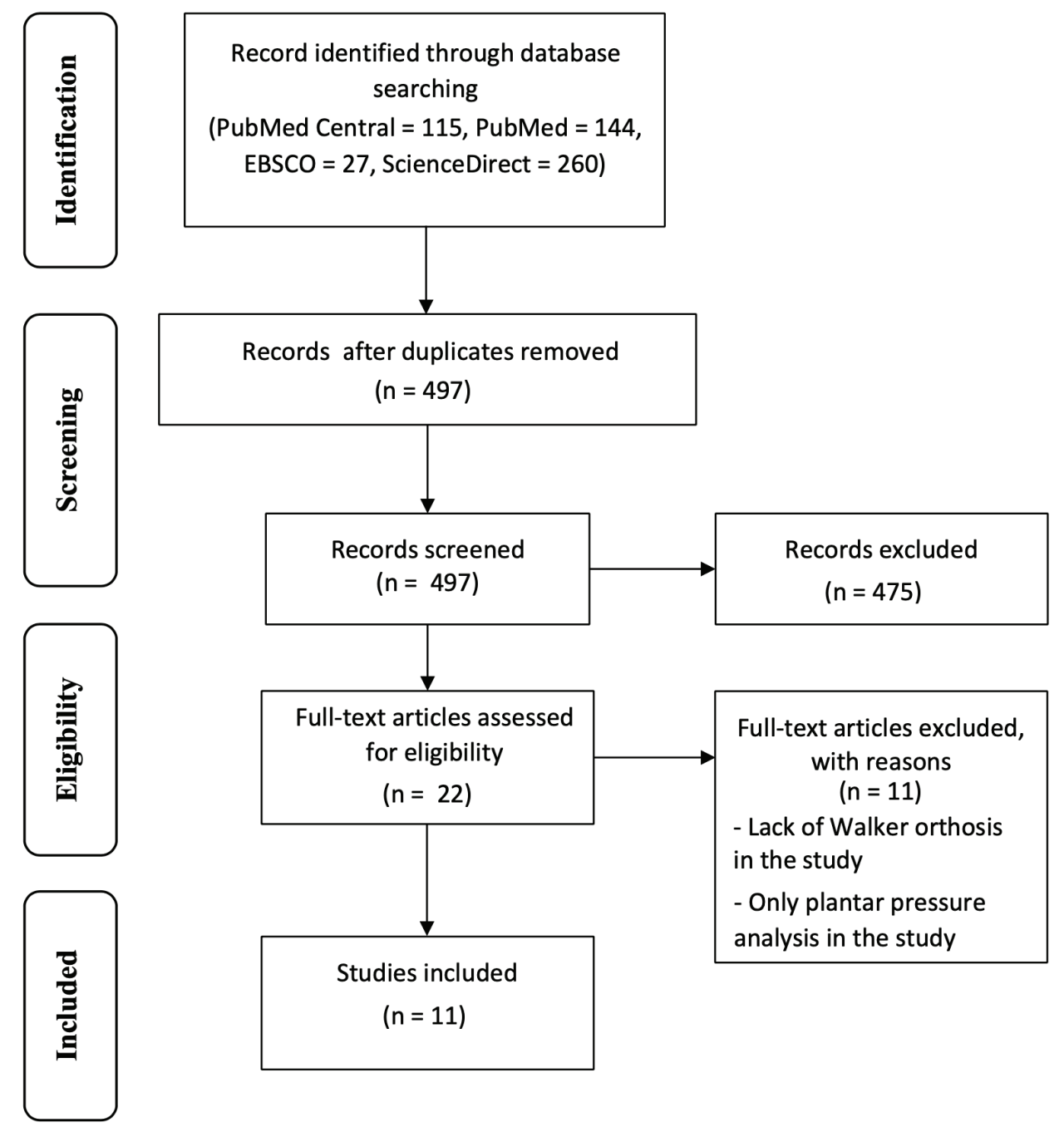

Fig. 1. Flowchart demonstrating the selection of articles through the review process 


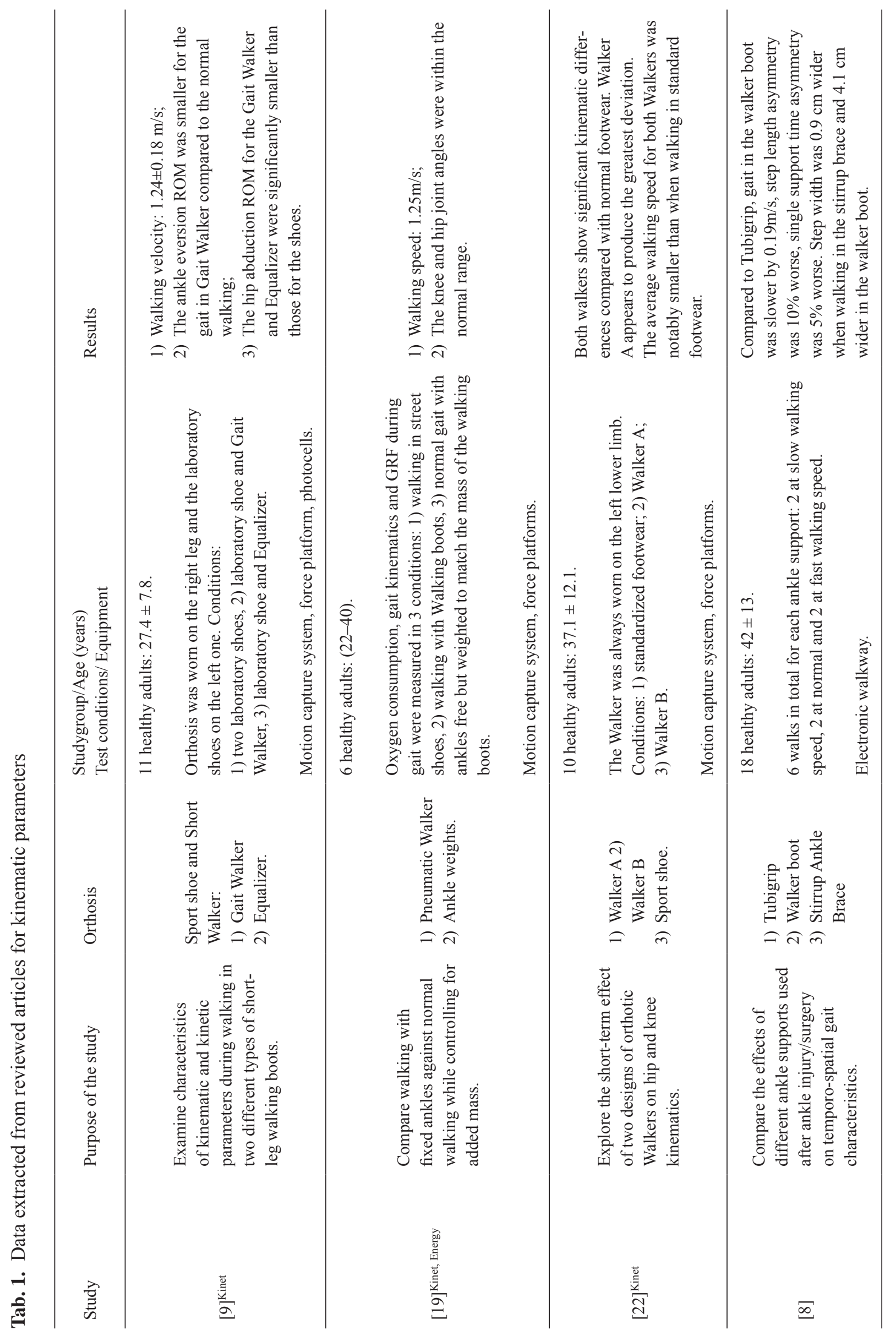




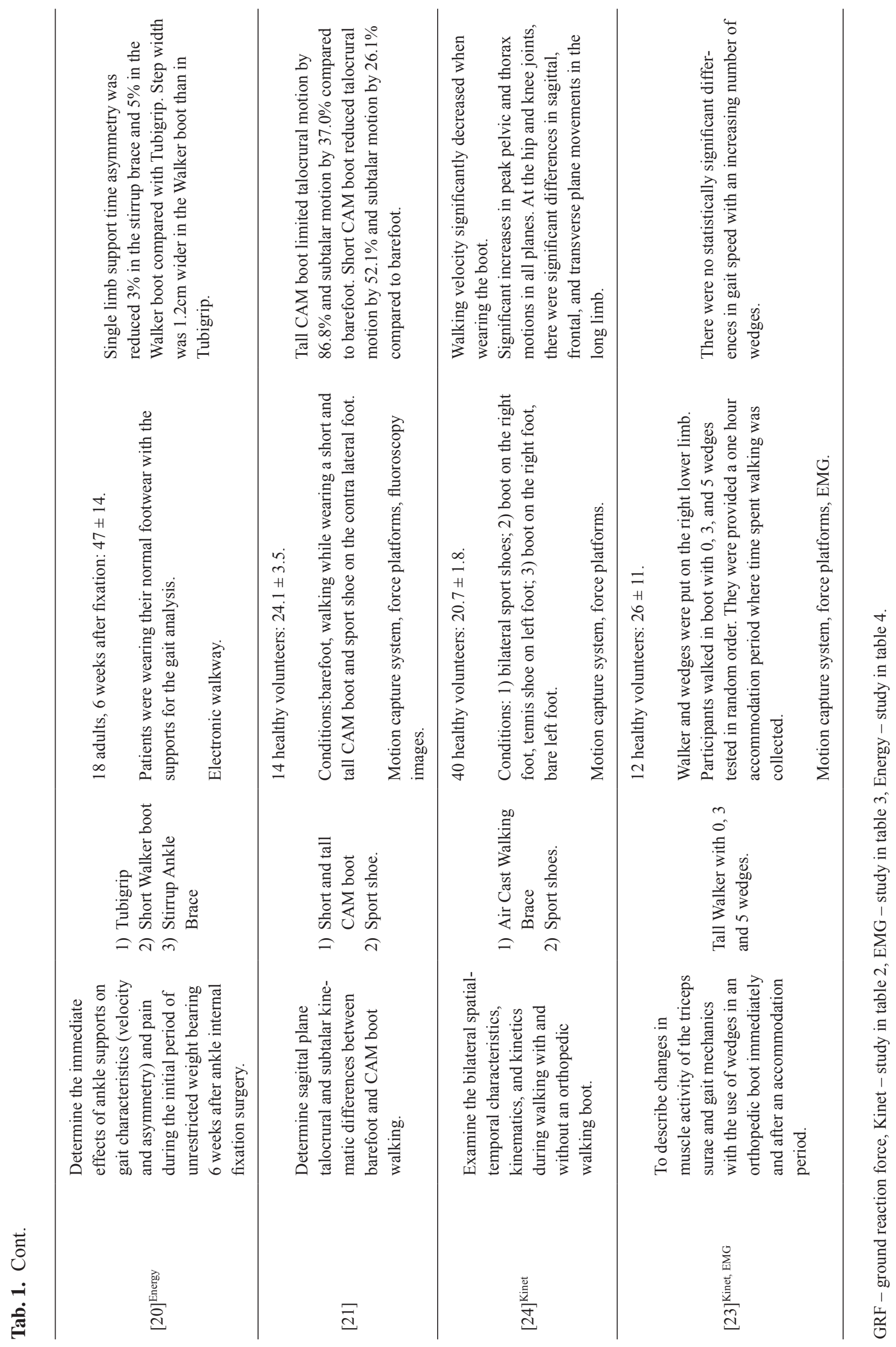


A Walker orthosis significantly decreases the range of motion in the ankle joint in the frontal and sagittal planes during fluoroscopy testing [21]. It also alters the range of motion during the knee transfer, involving a large amount of rotation in the hip joint [19]. In general, hip and knee joints of the leg without the orthosis are less or even not affected in the case of a range of movements in the frontal and sagittal planes. The biggest changes are observed in the opposite leg in the hip, knee and, as one study suggested, changes might also be seen in the opposite ankle joint.

\section{Kinetics parameters}

Six papers discussed how a Walker boot may affect kinetic parameters, but only one was designed just to assess ground reaction force (GRF) (Tab. 2). Five of these studies also analyzed kinematic parameters, one of them evaluated energy expenditure and one analyzed muscle activity.
Two studies [23,25] analyzed the effect of heel wedges on gait kinetics. Ground reaction forces were analyzed in four articles [9,23-25], whereas joint moments were analyzed in five [9,19,22-24]. Zhang, Clowers [9] and Keefer, King [25] showed that wearing Walker may cause a small initial peak in the vertical ground reaction force. The peak vertical ground reaction force was at the same level [9] or slightly but significantly (2-3\%) [24] lower compared to shoe walking depending on the research. The peak knee extension torque on the Walker limb were elevated (19-29\%) in every study addressing this issue. On the other hand, demand on the hip extensors was lower by $6 \%[9,22,24]$. Knee and hip abduction torques for the same limb were decreased $[9,24]$.

\section{Muscle activity}

Only two papers discussed how a Walker boot may affect muscle activity (Tab. 3). One of them was

Tab. 2. Data extracted from reviewed articles for kinetic parameters

\begin{tabular}{lll}
\hline Study & $\begin{array}{l}\text { Purpose of the } \\
\text { study }\end{array}$ & $\begin{array}{l}\text { Studygroup/ age (years) } \\
\text { Test conditions/ Equipment }\end{array}$
\end{tabular}

10 healthy volunteers: $22.6 \pm 1.68$.

Examine how

heel height differences in the walker and

shoe side may

influence GRFs

when wearing

a short walker.
Six conditions: lab shoes, gait Short Walkers: walker, gait walker with heel insert

1) Gait Walker on shoe side, gait walker with insert

2) Equalizer

3) Sport shoe on walker side, equalizer walker, equalizer walker with heel insert on shoe side.
The application of a walker created peak vertical and AP GRF prior to the normal peaks associated with the loading response. Peak propulsive AP GRF were smaller in all walker conditions compared to shoe on walker side.

Force platforms, photocells.

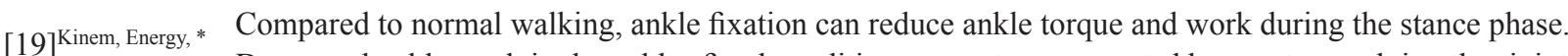
Decreased ankle work in the ankles fixed condition was not compensated by greater work in other joints.

Significant differences in peak knee extension torques were seen between all conditions with Walker A showing the highest knee extension torques followed by Walker B, and in the peak knee flexion torques

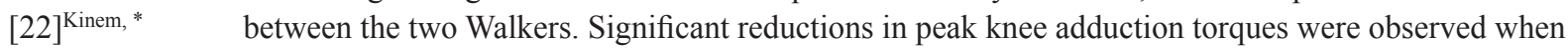
walking with both Walkers compared with sport footwear. Hip extension moments showed significant differences between Walker A and both Walker B and sport footwear.

Peak vertical GRF were significantly decreased on long limb. Peak AP GRF differed significantly across

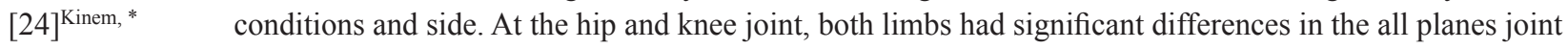
moments with the exception of the short limb frontal plane moment.

[23] Kinem, EMG, * Vertical GRF significantly decreased and peak knee extension power significantly increased with increasing number of wedges.

GRF - ground reaction force, AP - anterio-posterior, Kinem - study in table 1, EMG - study in table 3, Energy - study in table 4., * - purpose of the study, type of the orthosis, study group and test conditions/ equipment are contained in the table 1. 
Tab. 3. Data extracted from reviewed articles for EMG

\begin{tabular}{|c|c|c|c|c|}
\hline Study & $\begin{array}{l}\text { Purpose of the } \\
\text { study }\end{array}$ & Orthosis & $\begin{array}{l}\text { Studygroup/ age (years) } \\
\text { Test conditions/ Equipment }\end{array}$ & Results \\
\hline [17] & $\begin{array}{l}\text { Determine if } \\
\text { muscle activity } \\
\text { reduction is } \\
\text { similar using } \\
\text { a fiberglass } \\
\text { cast versus } \\
\text { a prefabricated } \\
\text { boot. }\end{array}$ & $\begin{array}{l}\text { Three } \\
\text { conditions: } \\
\text { 1) Barefoot; } \\
\text { 2) Fiberglass } \\
\text { cast } \\
\text { 3) High Walker }\end{array}$ & $\begin{array}{l}12 \text { healthy adults. } \\
\text { Subjects walked at their self- } \\
\text { selected speed for } 10 \text { trials in } \\
\text { each condition. } \\
\text { Force plate, EMG: left medial } \\
\text { gastrocnemius, lateral soleus, } \\
\text { and peroneal muscle. }\end{array}$ & $\begin{array}{l}\text { A significant decrease in muscle } \\
\text { activity was found between the } \\
\text { barefoot and boot conditions } \\
\text { for: gastrocnemius, soleus and } \\
\text { peroneals. } \\
\text { The comparison between } \\
\text { barefoot and cast established } \\
\text { a significant decrease in soleus } \\
\text { and peroneal activity. The boot } \\
\text { and cast comparison exhibited } \\
\text { a significant decrease in the gas- } \\
\text { trocnemius activity in the boot } \\
\text { compared with the cast. }\end{array}$ \\
\hline$[23]^{\text {Kinem, Kinet, * }}$ & increasing nur & of wedges. & $d$ & gnificantly decreased with the \\
\hline
\end{tabular}

Kinem - study in table 1 , Kinet - study in table $2,{ }^{*}$ - purpose of the study, type of the orthosis, study group and test conditions/ equipment are contained in the table 1 .

designed strictly to assess muscle activity and the other one analyzed kinematic and kinetic parameters.

Electromyography was performed in two papers. One of them [17] was designed specifically to analyze muscle activity while walking in a cast and a Walker boot. Overall muscle activity decreased by $20 \%$ in the Walker boot. The effect of the number of heel wedges on triceps surae activity was analyzed in the second article [23]. The five wedges significantly decreased medial gastrocnemius and soleus muscle activity, while 3 wedges decreased only soleus activity. Both articles tested groups of only 12 participants. Heel lifting and ankle immobilization are effective in decreasing the lower leg muscle activity.

\section{Energy cost and pain assessment}

Three papers discussed how a Walker boot may affect energy cost, pain and balance (Tab. 4). Energy expenditure of gait in a Walker boot was analyzed in one article [19]. Assessment of balance in a Walker boot was also performed in one article [26]. Furthermore, pain assessment was conducted also in one study. It concerned pain-relieving properties of different types of orthoses [20].

According to Vanderpool, Collins [19], decreased plantarflexion caused by ankle immobilization is compensated by a curved rocker bottom surface. It is possible that higher energy expenditure results from an added weight of Walker rather than the ankle immobilization. A Walker orthosis has also an effect on postural balance, but the effect of heel wedges on postural balance is inconclusive.

\section{Discussion}

This review aimed to summarize and update information on the currently published research explicitly related to the application of ankle-foot Walker orthoses and to help improve the understanding of how this type of orthoses influence gait pattern, in particular the kinematics, kinetics, and muscle activity parameters. To our knowledge no systematic reviews have been conducted in this topic.

One of the main goals of the rehabilitation of patients who have undergone a surgery or have been injured in the foot and ankle area is to protect and immobilize this zone and then to restore the normal gait pattern, which can be disturbed asymmetrically due to the unilateral presence of injury. Factors that may affect asymmetry during gait include muscle weakness, pain, proprioception disorder, movement range limitation, and no full load capacity $[28,29]$. The main function of an orthosis is to enable walking, relieve damaged tissue, limit the range of motion, and help reduce pain [8]. Compared to a total contact cast, an orthosis has many advantages: 
Tab. 4. Data extraction from reviewed articles for energy expenditure, balance and pain assessment

\begin{tabular}{|c|c|c|c|c|}
\hline Study & Purpose of the study & Orthosis & $\begin{array}{l}\text { Study group/ age (years) } \\
\text { Test conditions/ Equipment }\end{array}$ & Results \\
\hline$[19]^{\text {Kinem, Kinet, * }}$ & \multicolumn{4}{|c|}{$\begin{array}{l}\text { Gait in walking boots caused the total rate of energy expenditure for walking to increase significantly by } \\
4.1 \% \text { compared to normal shoes but differed by an insignificant amount }(0.4 \%) \text { compared to walking with } \\
\text { equivalent ankle weight. }\end{array}$} \\
\hline [26] & $\begin{array}{l}\text { Determine if a walking } \\
\text { boot increases body } \\
\text { motion during balance } \\
\text { tests across a range of } \\
\text { simple to challenging } \\
\text { conditions and if adding } \\
\text { a heel lift to the non- } \\
\text { involved limb would } \\
\text { reduce body motion by } \\
\text { correcting the leg length } \\
\text { discrepancy. }\end{array}$ & Walking boot & $\begin{array}{l}\text { In each test, subjects were wearing sport } \\
\text { shoes, a walking boot, or a walking boot } \\
\text { and a heel lift in the contralateral shoe. } \\
\text { Quiet stance test was performed during } \\
\text { standing with both eyes open and } \\
\text { closed, on rigid and soft surfaces. }\end{array}$ & $\begin{array}{l}\text { Walking boot } \\
\text { significantly affected } \\
\text { the balance in each } \\
\text { test. When wearing } \\
\text { the wedge, the test } \\
\text { result in quiet stance } \\
\text { was significantly } \\
\text { better than when } \\
\text { wearing the orthosis } \\
\text { alone, but in the other } \\
\text { tests, no differences } \\
\text { were noticed. }\end{array}$ \\
\hline$[20]^{\text {Kinem, * }}$ & \multicolumn{4}{|c|}{$\begin{array}{l}\text { Test conditions/equipment: VAS scale for pain assessment. } \\
\text { The pain was significantly lower in the Walker boot and to a lesser extent in the stirrup brace compared } \\
\text { with Tubigrip. }\end{array}$} \\
\hline
\end{tabular}

Kinem - study in table 1 , Kinet - study in table $2, *$ - purpose of the study, type of the orthosis, study group and test conditions/ equipment are contained in the table 1 .

it is lighter, has a smaller effect on gait biomechanics and can be removed to maintain tissue hygiene and to perform exercises [6,9].

It is proved that leg length discrepancy alters the gait kinetics and kinematics and may lead to conditions such as low back pain, plantar fasciitis and osteoarthritis of the knee and hip joints. In their clinical practice, many physiotherapists and physicians recommend that their patients wear an athletic shoe on the opposite leg to the one with a Walker orthosis. This suggestion was partially proven right. Mieras, Singleton [30] demonstrated that wearing an athletic shoe on the leg opposite to that with a Walker orthosis significantly decreases peak plantar pressure in the rear foot area compared to the barefoot condition. In another study, Gulgin, Hall [24] tested walking in Walker on the left foot and shoe or barefoot on the right. They did not conclude if wearing a shoe on the contra lateral leg might be beneficial.

The changes in the center of pressure during the use of a walker orthosis were examined by Lajevardi-Khosh, Bamberg [31] and North, Potter [32]. Both studies yielded different results. North, Potter [32], who tested healthy participants, suggested that the center of pressure shifts anteriorly while the weight bearing increases. On the other hand, Lajevardi-Khosh, Bamberg [31] showed that the center of pressure shifts posteriorly in lower leg fracture patients towards heel during recovery. The distinction may exist because healthy and recovering participants performed partial weight-bearing ambulation differently.

Muscle activity was analyzed in only two articles. It was proved by Kadel et al. [17] that a high-cut Walker may be used instead of a custom cast if the goal during the treatment is to limit muscle activity of the lower leg muscles. It significantly decreases overall muscle activity compared to barefoot walking and significantly decreases gastrocnemius muscle activity compared to the custom cast. Those differences between walker and the cast may be caused by the rocking bottom of the walker. Richards, Payne [22] proved that even subtle differences in the Walker design may have a significant effect on knee joint kinetics and kinematics. Zellers, Tucker [23] showed that with an increasing number of heel wedges, muscle activity in the gastrocnemius and soleus muscles decreases. In both studies, surface electrodes were placed on the skin under the walker orthosis. In such conditions, even the slightest compression on an electrode may alter the measurement.

Gait kinematics has been analyzed in only a few studies. Zhang, Clowers [9] showed no significant changes in ankle angle in the sagittal plane between a low-cut Walker or Equalizer and a shoe condition. On the contrary, McHenry, Exten [21] using the fluoroscopy unit showed that low-cut and high-cut Walkers significantly 
decrease movement in the sagittal plane, with better outcomes obtained for the high-cut Walker. During the analysis of kinetics, Gulgin, Hall [24] found a significant difference in peak vertical ground reaction forces in contrast to Zhang, Clowers [9]. Also, they found significant decreases in anterior and posterior ground reaction forces. In both studies, the researchers found increased internal knee extensor torques in the lower limb. Higher forces acting on the limb with a walker may occur due to leg length discrepancy and may lead to knee pain in the longer limb. There have been attempts to decrease leg length discrepancy by putting an additional heel wedge into a shoe on the leg opposite to the walker. Keefer, King [25] used this method but failed to find differences in the initial peak between walking in shoes and walking with the heel wedge in the shoe and the walker on the opposite leg. Keene, Willett [8] compared three different types of orthoses. They found that a walker orthosis causes significantly greater gait disruptions than Tubigrip. Interestingly, they did not observe any important differences between a stirrup brace and Tubigrip although the stirrup brace is believed to be stiffer and provide stabilization in the frontal plane. They also suggested that a walker orthosis should be considered a primary choice six weeks after surgery due to its capability to reduce pain and increase the base of support.

Summarizing, the Walker orthosis was proved to alter gait biomechanics and it should be used reasonably. The analysis was carried out for kinematic and kinetic parameters, it appears that hip and knee joints of the leg with orthosis are those most affected. Changes are noticeable in all three planes during stance phase. Increased knee and hip flexion, knee internal rotation and hip adduction. What is more gait in the orthosis induces decrease of gait velocity and increase of step width. It was resulted that hip and knee joints of the leg without the orthosis are less or even not affected in the case of a range of movements in the frontal and sagittal planes.

It is worth mentioning that in the papers including healthy individuals the study subjects were wearing orthoses just for tests and were not using them for a longer period of time, whereas patients after surgeries were using them on average for 6-8 weeks and patients with foot ulcers in diabities mellitious for 6-10 weeks. The topic of wearing the orthosis for a long time was discussed only in one work [22]. Richards, Payne [22] showed that it may cause secondary site pain in the ipsilateral knee, contralateral hip and the lower back in $67 \%$ of patients and that a third of patients had ongoing pain 3 months after the transition to normal boot wear. On the other hand, we think that in our review it is justified to compare different applications of orthoses because all the participants were ambulating with full weight bearing without any additional support. It is our belief that this condition makes all gaits comparable, however it does not exclude the possibility that some differences might occur. On the other hand, we acknowledge the fact that in some instances we compared different types of Walker orthoses, which is one of the drawbacks of this article. Additionally, it is worth emphasizing that if the injured person does not feel pain and is not afraid to put weight on the limb in the orthosis, the gait parameters should not differ from the parameters of healthy people wearing an orthosis. Otherwise, the injured person may put more weight on the uninjured limb. After reviewing the literature, it is clear that the orthosis introduces changes in the biomechanics of gait in healthy people. Research [27] on the symptoms of people wearing orthoses for medical reasons has shown the emergence of new pain ailments. This means that the changes caused by the orthosis are greater in sick people or that similar changes in the biomechanics of the gait cause pain after a longer period of use of the orthosis. However, this is our assumption and the additional study on injured patients should be performed to verify this conjecture.

One limitation of the study is that most articles had a low number of participants. The groups studied were not coherent and they mostly included able-bodied people, which makes generalization of the patients impossible. Other limitations were that participants were walking at different walking speeds which were not always monitored. The types of used orthoses were also different and it was not always possible to verify whether the orthosis was a high-cut Walker or a low-cut one. In conclusion, this study is a comprehensive repository of the problem of the effect of Walker orthoses on gait biomechanics.

\section{Conclusions}

A Walker orthosis is a useful plantar pressure reliving device as long as the patient is willing to cooperate. It also allows earlier weightbearing and rehabilitation. Due to problems connected with LLD Walker, an orthosis should always be prescribed with a compensation of the LLD (eg. shoe) on the opposite leg because the patient may not be aware of the problem. What is more, a Walker orthosis should be worn for the shortest period of time possible due to LLD and ankle immobilization which may cause secondary site pain. Another drawback of wearing an orthosis is increased energy expenditure. It has been shown that further research concerning the influence of heel wedges in a Walker boot on gait biomechanics is required. Moreover, additional research should include patients and investigate the effect of ankle-foot orthoses on muscle force distribution, 
which corresponds to EMG. Orthosis pressuring electrodes may cause problems with obtaining reliable data from EMG. Muscle force distribution obtained by computer muscle simulation would be the method of choice to obtain reliable data. In conclusion, this study is a comprehensive repertory of the problem of the effect of Walker orthoses on gait biomechanics.

\section{Funding}

This work was supported by Józef Piłsudski University of Physical Education in Warsaw, Faculty of Rehabilitation, Scientific School "Diagnostics of locomotor system in selected dysfunctions as the basis for planning the rehabilitation process"

\section{Conflicts of interest}

The authors declare no conflict of interest.

\section{References}

1. Karimi MT, Kamali M, Fatoye F. Evaluation of the efficiency of cervical orthoses on cervical fracture: A review of literature. J Craniovertebr Junction Spine. 2016; 7(1): 13-9.

2. Katsuhira J, Matsudaira K, Oka H, et al. Efficacy of a trunk orthosis with joints providing resistive force on low back load during level walking in elderly persons. Clin Interv Aging. 2016; 11: 1589-97.

3. Pritchard K, Edelstein J, Zubrenic E, et al. Systematic review of orthoses for stroke-induced upper extremity deficits. Top Stroke Rehabil. 2019; 26(5): 389-98.

4. Parween R, Shriram D, Mohan RE, et al. Methods for evaluating effects of unloader knee braces on joint health: a review. Biomed Eng Lett. 2019; 9(2): 153-68.

5. Healy A, Farmer S, Eddison N, et al. A scoping literature review of studies assessing effectiveness and costeffectiveness of prosthetic and orthotic interventions. Disabil Rehabil Assist Technol. 2020; 15(1): 60-6.

6. Karkhanis S, Mumtaz H, Kurdy N. Functional management of Achilles tendon rupture: A viable option for non-operative management. Foot Ankle Surg. 2010; 16(2): 81-6.

7. Crenshaw SJ, Pollo FE, Brodsky JW. The effect of ankle position on plantar pressure in a short leg walking boot. Foot Ankle Int. 2004; 25(2): 69-72.

8. Keene DJ, Willett K, Lamb SE. The effects of ankle supports on gait in adults: A randomized cross-over study. J Electromyogr Kinesiol. 2015; 25(6): 973-81.

9. Zhang S, Clowers KG, Powell D. Ground reaction force and 3D biomechanical characteristics of walking in short-leg walkers. Gait Posture. 2006; 24(4): 487-92.

10. Hunt KJ, Goeb Y, Esparza R, et al. Site-specific loading at the fifth metatarsal base in rehabilitative devices: implications for Jones fracture treatment. PM R. 2014; 6(11): 1022-9.

11. Le M, Anderson R. Zone II and III fifth metatarsal fractures in athletes. Curr Rev Musculoskelet Med. 2017; 10(1): 86-93.

12. Wynter S, Grigg C. Lisfranc injuries. Aust Fam Physician. 2017; 46(3): 116-9.

13. White SC, Gilchrist LA, Wilk BE. Asymmetric limb loading with true or simulated leg-length differences. Clin Orthop Relat Res. 2004; (421): 287-92.

14. Imanishi J, Choong PF. Three-dimensional printed calcaneal prosthesis following total calcanectomy. Int J Surg Case Rep. 2015; 10: 83-7.

15. Mahmood S, Huffman LK, Harris JG. Limb-length discrepancy as a cause of plantar fasciitis. J Am Podiatr Med Assoc. 2010; 100(6): 452-5.

16. Harvey WF, Yang M, Cooke TD, et al. Association of leg-length inequality with knee osteoarthritis: a cohort study. Ann Intern Med. 2010; 152(5): 287-95.

17. Kadel NJ, Segal A, Orendurff M, et al. The efficacy of two methods of ankle immobilization in reducing gastrocnemius, soleus, and peroneal muscle activity during stance phase of gait. Foot Ankle Int. 2004; 25(6): 406-9.

18. Akizuki KH, Gartman EJ, Nisonson B, et al. The relative stress on the Achilles tendon during ambulation in an ankle immobiliser: implications for rehabilitation after Achilles tendon repair. Br J Sports Med. 2001; 35(5): 329-33.

19. Vanderpool MT, Collins SH, Kuo AD. Ankle fixation need not increase the energetic cost of human walking. Gait Posture. 2008; 28(3): 427-33.

20. Keene DJ, Willett K, Lamb SE. The Immediate Effects of Different Types of Ankle Support Introduced 6 Weeks After Surgical Internal Fixation for Ankle Fracture on Gait and Pain: A Randomized Crossover Trial. J Orthop Sports Phys Ther. 2016; 46(3): 157-67.

21. McHenry BD, Exten EL, Cross JA, et al. Sagittal Subtalar and Talocrural Joint Assessment During Ambulation With Controlled Ankle Movement (CAM) Boots. Foot Ankle Int. 2017; 38(11): 1260-6.

22. Richards J, Payne K, Myatt D, et al. Do orthotic walkers affect knee and hip function during gait? Prosthet Orthot Int. 2016; 40(1): 137-41.

23. Zellers JA, Tucker LA, Higginson JS, et al. Changes in gait mechanics and muscle activity with wedge height in an orthopaedic boot. Gait Posture. 2019; 70: 59-64.

24. Gulgin H, Hall K, Luzadre A, et al. 3D gait analysis with and without an orthopedic walking boot. Gait Posture. 2018; 59: 76-82.

25. Keefer M, King J, Powell D, et al. Effects of modified short-leg walkers on ground reaction force characteristics. Clin Biomech. 2008; 23(9): 1172-7. 
26. Goodworth AD, Kunsman M, Depietro V, et al. Characterization of how a walking boot affects balance. Journal of Prosthetics and Orthotics. 2014; 26(1): 54-60.

27. Ready LV, Fisk EG, Ciurylo W, et al. Associated Joint Pain With Controlled Ankle Movement Walker Boot Wear. J Am Acad Orthop Surg Glob Res Rev. 2018; 2(12): e044.

28. Lin CW, Hiller CE, de Bie RA. Evidence-based treatment for ankle injuries: a clinical perspective. J Man Manip Ther. 2010; 18(1): 22-8.

29. O'Connor SR, Bleakley CM, Tully MA, et al. Predicting functional recovery after acute ankle sprain. PLoS One. $2013 ; 8(8)$ : e72124.
30. Mieras JN, Singleton TJ, Barrett SL. Contralateral peak plantar pressures with a postoperative boot: a preliminary study. J Am Podiatr Med Assoc. 2011; 101(2): 127-32.

31. Lajevardi-Khosh A, Bamberg S, Rothberg D, et al. Center of pressure in a walking boot shifts posteriorly in patients following lower leg fracture. Gait Posture. 2019; 70: 218-21.

32. North K, Potter MQ, Kubiak EN, et al. The effect of partial weight bearing in a walking boot on plantar pressure distribution and center of pressure. Gait Posture. 2012; 36(3): 646-9. 